\title{
Development of family of artificial neural networks for the prediction of cutting tool condition
}

\author{
Spaić, O. ${ }^{a}$, Krivokapić, Z. ${ }^{\mathrm{b}}$, Kramar, D. ${ }^{\mathrm{c},}$ \\ ${ }^{a}$ University in East Sarajevo, Faculty of Production and Management Trebinje, Bosnia and Herzegovina \\ buniversity in Montenegro, Faculty of Mechanical Engineering Podgorica, Montenegro \\ ${ }^{c}$ University in Ljubljana, Faculty of Mechanical Engineering Ljubljana, Slovenia
}

\begin{abstract}
A B S T R A C T
Recently, besides regression analysis, artificial neural networks (ANNs) are increasingly used to predict the state of tools. Nevertheless, simulations trained by cutting modes, material type and the method of sharpening twist drills (TD) and the drilling length from sharp to blunt as input parameters and axial drilling force and torque as output ANN parameters did not achieve the expected results. Therefore, in this paper a family of artificial neural networks (FANN) was developed to predict the axial force and drilling torque as a function of a number of influencing factors. The formation of the FANN took place in three phases, in each phase the neural networks formed were trained by drilling lengths until the drill bit was worn out and by a variable parameter, while the combinations of the other influencing parameters were taken as constant values. The results of the prediction obtained by applying the FANN were compared with the results obtained by regression analysis at the points of experimental results. The comparison confirmed that the FANN can be used as a very reliable method for predicting tool condition.
\end{abstract}

(c) 2020 CPE, University of Maribor. All rights reserved.

\section{ARTICLE INFO}

Keywords:

Drilling;

Cutting tool;

Twist drill bits;

Axial force;

Tool wear;

Prediction;

Artificial neural networks;

Back propagation

*Corresponding author:

davorin.kramar@fs.uni-lj.si

(Kramar, D.)

Article history:

Received 8 January 2020

Revised 24 June 2020

Accepted 27 June 2020

\section{References}

[1] Spaić, O., Krivokapić, Z., Ivanković R. (2013). Mathematical modelling of cutting force as the most reliable information bearer on cutting tools wearing phenomenon, Journal of Mechanics Engineering and Automation (JMEA), Vol. 3, No. 12, 772-777.

[2] Krivokapić, Z., Zogović, V., Spaić O. (2006). Using neural netvorks to follow the wear of a 390 twist drill, Strojniški vestnik - Journal of Mechanichal Engineering, Vol. 52, No. 7-8, 437-442.

[3] Kaya, B., Oysu, C., Ertunc, H.M. (2011). Force-torque based on-line tool wear estimation system for CNC milling of Inconel 718 using neural networks, Advances in Engineering Software, Vol. 42, No. 3, 76-84, doi: 10.1016/ j.advengsoft.2010.12.002.

[4] Wu, D., Jennings, C., Terpenny, J., Gao, R.X., Kumara, S. (2017). A comparative study on machine learning algorithms for smart manufacturing: Tool wear prediction using random forests, Journal of Manufacturing Science and Engineering, Vol. 139, 071018-1-071018-9, doi: 10.1115/1.4036350.

[5] Sekulic, M., Pejic, V., Brezocnik, M., Gostimirović, M., Hadzistevic, M. (2018). Prediction of surface roughness in the ball-end milling process using response surface methodology, genetic algorithms, and grey wolf optimizer algorithm, Advances in Production Engineering \& Management, Vol. 13, No. 1, 18-30, doi: 10.14743/apem2018. $\underline{1.270 .}$.

[6] Tamang, S.K.; Chandrasekaran, M. (2015). Modeling and optimization of parameters for minimizing surface roughness and tool wear in turning $\mathrm{Al} / \mathrm{SiCp} \mathrm{MMC}$, using conventional and soft computing techniques, Advances in Production Engineering \& Management, Vol. 10, No. 2, 59-72, doi: 10.14743/apem2015.2.192.

[7] Neto, F.C., Gerônimo, T.M., Cruz, C.E.D., Aguiar, P.R., Bianchi, E.E.C. (2013). Neural models for predicting hole diameters in drilling processes, Procedia CIRP, Vol. 12, 49-54, doi: 10.1016/i.procir.2013.09.010. 
[8] Rao, K.V., Murthy, B.S.N., Rao, N.M. (2014). Prediction of cutting tool wear, surface roughness and vibration of work piece in boring of AISI 316 steel with artificial neural network, Measurement, Vol. 51, 63-70, doi: 10.1016/ i.measurement.2014.01.024.

[9] Martins, C.H.R., Aguiar, P.R., Frech, A., Bianchi, E.C. (2014). Tool condition monitoring of single-point dresser using acoustic emission and neural networks models, IEEE Transactions on Instrumentation and Measurement, Vol. 63, No. 3, 667-679, doi: 10.1109/TIM.2013.2281576.

[10] Kannan, T.D.B., Kannan, G.R., Umar, M., Kumar, S.A. (2015). ANN approach for modelling parameters in drilling operation, Indian Journal of Science and Technology, Vol. 8, No. 22, doi: 10.17485/ijst/2015/v8i22/79097.

[11] Benkedjouh, T., Medjaher, K., Zerhouni, N., Rechak, S. (2015). Health assessment and life prediction of cutting tools based on support vector regression, Journal of Intelligent Manufacturing, Vol. 26, No. 2, 213-223, doi: $10.1007 / \mathrm{s} 10845-013-0774-6$.

[12] Drouillet, C., Karandikar, J., Nath, C. , Journeaux, A.-C., El Mansori, M., Kurfess, T. (2016): Tool life predictions in milling using spindle power with the neural network technique, Journal of Manufacturing Processes, Vol 22, 161168, doi: 10.1016/i.jmapro.2016.03.010.

[13] D'Addona, D.M., Matarazzo, D., de Aguiar, P.R., Bianchi, E.C., Martins, C.H.R. (2016). Neural networks tool condition monitoring in single-point dressing operations, Procedia CIRP, Vol. 41, 431-43, doi: org/10.1016/i.procir. 2016.01.001.

[14] Patra, K., Jha, A.K., Szalay, T., Ranjan, J., Monostori, L. (2017). Artificial neural network based tool condition monitoring in micro mechanical peck drilling using thrust force signal, Precision Engineering, Vol. 48, 279-291, doi: org/10.1016/i.precisioneng.2016.12.011.

[15] Khorasani, A.M., Yazdi, M.R.S. (2017). Development of a dynamic surface roughness monitoring system based on artificial neural networks (ANN) in milling operation, The International Journal of Advanced Manufacturing Technology, Vol. 93, 141-151, doi: 10.1007/s00170-015-7922-4.

[16] Mikołajczyk, T., Nowicki, K., Bustillo, A., Yu Pimeno, D. (2018). Predicting tool life in turning operations using neural networks and image processing, Mechanical Systems and Signal Processing, Vol. 104, 503-513, doi: 10.1016/i.ymssp.2017.11.022.

[17] Wang, Q., Jia, X. (2020). Multi-objective optimization of CFRP drilling parameters with a hybrid method integrating the ANN, NSGA-II and fuzzy C-means, Composite Structures, Vol. 235, 111803, doi: 10.1016/i.compstruct. 2019.111803.

[18] Kumar, R., Hynes, N.R.J. (2020). Prediction and optimization of surface roughness in thermal drilling using integrated ANFIS and GA approach, Engineering Science and Technology, an International Journal, Vol. 23, Vol. 1, 3041, doi: 10.1016/i.jestch.2019.04.011.

[19] Mondal, N., Mandal, S., Mandal, M.C. (2020). FPA based optimization of drilling burr using regression analysis and ANN model, Measurement, Vol. 152, 107327, doi: 10.1016/i.measurement.2019.107327.

[20] Schorr, S., Möller, M., Heib, J., Bähre, D. (2020). Quality prediction of drilled and reamed bores based on torque measurements and the machine learning method of random forest, Procedia Manufacturing, Vol. 48, 894-901, doi: 10.1016/i.promfg.2020.05.127.

[21] Yin, C.P., Wu, Z.P., Dong, Y.W., You, Y.C., Liao, T. (2019). Femtosecond laser helical drilling of nickel-base singlecrystal super-alloy: Effect of machining parameters on geometrical characteristics of micro-holes, Advances in Production Engineering \& Management, Vol. 14, No. 4, 407-420, doi: 10.14743/apem2019.4.337.

[22] Spaić, 0. (2017). Teorija rezanja, Univerzitet u Istočnom Sarajevu, Fakultet za proizvodnju i menadžment Trebinje, Trebinje, Bosnia and Herzegovina. 


\title{
Razvoj družine umetnih nevronskih mrež za napovedovanje stanja rezalnega orodja
}

\author{
Spaić, O. ${ }^{a}$, Krivokapić, Z. ${ }^{b}$, Kramar, D. ${ }^{c,}{ }^{*}$ \\ aniversity in East Sarajevo, Faculty of Production and Management Trebinje, Bosnia and Herzegovina \\ ${ }^{b}$ University in Montenegro, Faculty of Mechanical Engineering Podgorica, Montenegro \\ 'University in Ljubljana, Faculty of Mechanical Engineering Ljubljana, Slovenia
}

\begin{abstract}
POVZETEK
$\mathrm{V}$ zadnjem času se za napovedovanje stanja orodij poleg regresijske analize vse bolj uporabljajo umetne nevronske mreže (ANN). Kljub temu simulacije ANN, naučenih na različnih načinih rezanja, vrstah materiala, oblikah vijačnega svedra (TD) in dolžinah rezalnega roba svedra za napoved osne vrtalne sile in navora, niso dosegle pričakovanih rezultatov. Zato je bila v tem prispevku razvita družina umetnih nevronskih mrež (FANN) za napovedovanje osne sile in navora pri vrtanju v odvisnosti od številnih vplivnih dejavnikov. Oblikovanje FANN je potekalo $v$ treh fazah. $V$ vsaki fazi so se nastale nevronske mreže učile na vrtalni dolžini, dokler se sveder ni obrabil in na spremenljivem parametru, medtem ko so bile kombinacije drugih vplivnih parametrov nespremenljive. Rezultate napovedi, pridobljene $\mathrm{z}$ uporabo FANN, smo primerjali z rezultati, pridobljenimi z regresijsko analizo v točkah, pridobljenih s pomočjo eksperimentalnih rezultatov. Primerjava je potrdila, da je FANN mogoče uporabiti kot zanesljivo metodo za napovedovanje stanja orodja.

(c) 2020 CPE, University of Maribor. All rights reserved.
\end{abstract}

\begin{tabular}{l} 
P O D A T K I O Č L A N K U \\
\hline Ključne besede: \\
Vrtanje; \\
Rezalno orodje; \\
Svedri za vrtanje; \\
Osna sila; \\
Obraba orodja; \\
Napoved; \\
Umetne nevronske mreže; \\
Vzvratno razširjanje \\
*Kontaktna oseba: \\
davorin.kramar@fs.uni-lj.si \\
(Kramar, D.) \\
Zgodovina članka: \\
Prejet 8. januarja 2020 \\
Popravljen 24. junija 2020 \\
Sprejet 27. junija 2020
\end{tabular}

